\title{
Alzheimer's disease risk factors as mediators of subjective memory impairment and objective memory decline: protocol for a construct-level replication analysis
}

Nikki L. Hill ${ }^{*}$ a and Jacqueline Mogle

\begin{abstract}
Background: Subjective memory impairment (SMI), or the perception of memory problems in the absence of objective memory deficits, is associated with negative outcomes of individual and societal significance, including a substantially increased risk of Alzheimer's disease (AD). However, little is known regarding the mediators that link SMI and memory decline in some individuals, or which older adults with SMI are at greatest risk for memory decline. In this study, we will examine modifiable AD risk factors (specifically affective symptoms and activity participation) as mediators underlying linkages among SMI and memory decline over time; furthermore, we will characterize SMI subgroups at highest risk for memory decline via this pathway.
\end{abstract}

Methods: This study utilizes a series of construct-level replication analyses across four large longitudinal datasets to maximize the unique aspects of each dataset as well as test the reproducibility of findings across multiple populations to establish generalizability. The current study's sample $(n>40,000)$ is drawn from the Einstein Aging Study, Health and Retirement Study, Minority Aging Research Study, and National Health and Aging Trends Study. Participants must meet the following basic criteria for inclusion: age 55 or older and no evidence of cognitive impairment at baseline. We will use multilevel modeling to determine whether higher levels of SMI are related to increased affective symptoms and decreased activity participation, as well as whether this relationship is moderated by neuroticism, family history of AD, and race/ethnicity. Finally, we will test our full conceptual model that examines whether changes in affective symptoms and activity participation mediate the relationship between SMI and objective memory decline. Specifically, we will test moderated mediation as we hypothesize these relationships to hold among subgroups of older adults.

Discussion: Discovery of modifiable AD risk factors that mediate the association between SMI and memory decline (the earliest and most central deficit in AD) will provide explicit, and potentially novel, targets for intervention. Additionally, identifying individuals at highest risk for negative reactions to SMI will serve to enrich samples for future research as well as to help guide the development of SMI assessment tools to identify older adults at greatest risk for debilitating outcomes.

Keywords: Cognition, Memory, Self-report, Subjective memory, Alzheimer's disease

\footnotetext{
* Correspondence: nikki.hill@psu.edu

College of Nursing, The Pennsylvania State University, 201 Nursing Sciences

Bldg, University Park, PA 16802, USA
}

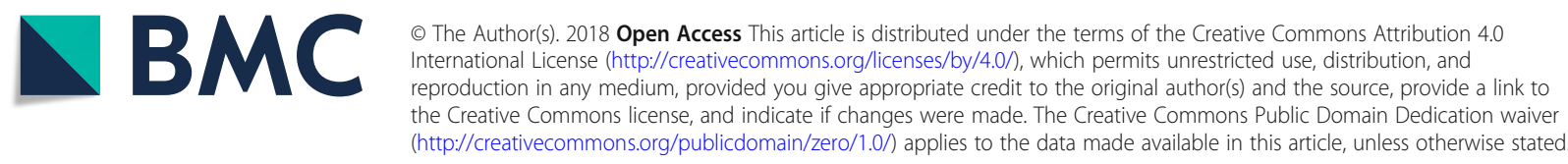




\section{Background}

Alzheimer's disease (AD) is, by definition, a progressive disease with an insidious onset. In its preclinical phase, pathological changes accumulate for a decade or more prior to clinical presentation of cognitive deficits [1]. Subjective memory impairment (SMI), the report of memory impairment with no clinical indication of memory deficit [2], often precedes clinically identifiable memory deficit early in the course of AD [3]. Affected individuals become aware of functional changes that may be too subtle for clinical testing to detect, or are most obvious in complex, real-world environments [46]. Over $20 \%$ of community-dwelling older adults experience SMI [7, 8], and this group is up to four times as likely to develop mild cognitive impairment or $\mathrm{AD}$ within the next 7 years [9]. However, given that SMI does not always lead to $\mathrm{AD}$, there is much we do not yet understand regarding SMI as a preclinical indicator of actual memory decline. Little is known regarding which individuals with SMI are at highest risk for developing $\mathrm{AD}$ or what individual factors influence the trajectory of cognitive decline [10].

Beyond an increased AD risk, SMI is associated with decreased participation in cognitive, physical, and social activities [11-14] as well as higher levels of affective (depressive or anxiety) symptoms [15]. Known AD risk factors include non-modifiable factors such as low educational attainment and genetic predisposition, but also modifiable factors such as depression, physical inactivity, lack of social connectedness, and a less cognitively complex lifestyle [16-18]. Affective symptoms and lower physical, cognitive, and social activity participation are known to co-occur with SMI and may result from negative reactions to the experience of SMI. For example, concerns about memory decline may precipitate depressive symptoms $[19,20]$ or withdrawal from cognitively protective behaviors such as participating in social activities [21]. Understanding these relationships is of critical importance since affective symptoms and lower activity participation are implicated in increased AD risk. Furthermore, older adults' reactions to SMI may influence mental health (i.e., psychological mechanisms such as affective symptoms) and lifestyle (i.e., behavioral mechanisms such as decreases in activity participation). Depending on whether these reactions are positive (e.g., proactively increasing participation in cognitively complex activities leading to neuroprotective and compensatory effects; [22]), or negative (e.g., increased memoryrelated anxiety leading to the deleterious cognitive effects of chronic stress; [23]) the timeline of clinically identifiable memory deficit along the AD trajectory may be altered.

Evidence suggests that reactions to SMI in older adults are influenced by several individual factors. First, the personality trait of neuroticism (i.e., a persistent tendency to experience negative emotions) is associated with SMI in community [24, 25] and memory clinic [26] samples. Complicating our understanding of neuroticism's role in the experience of SMI is the increased risk for individuals higher in neuroticism to experience affective symptoms (known risk factors for AD). Longitudinal studies suggest that individuals higher in neuroticism may be more attuned to notice memory problems or less likely to habituate to memory changes [27], and that neuroticism may moderate associations between SMI and psychological as well as physical health [28]. Second, a family history of AD or other dementias is associated with increased worry about SMI [29]. Fear of developing AD is common among older adults, and its impact is influenced by personal proximity to the disease: a family history of AD and/or experience as an informal caregiver to a family member with AD increases older adults' AD-related fears [30]. Third, lower levels of AD knowledge among Blacks compared to Whites [31] as well as $\mathrm{AD}$ diagnoses occurring at more advanced disease stages in Blacks [32], suggest that perceptions of SMI may differ among racial or ethnic groups. Therefore, individual characteristics are likely important to consider when examining relationships among SMI, modifiable AD risk factors, and memory decline over time.

Complicating the interpretation of existing evidence regarding SMI is the heterogeneity of assessment approaches across studies. These range from single-item measures to extensive symptom checklists, self-ratings of memory abilities to endorsements of memory concerns, and comparisons to one's self over time to comparisons with others [33]. In their review of subjective cognitive decline measures employed in preclinical AD investigations, Rabin and colleagues urge caution in comparing findings across studies due to the substantial heterogeneity of measures [34]. There is an identified need to interpret findings that associate SMI and adverse outcomes with specificity: item construction matters.

The experience of SMI is associated with negative outcomes of great individual and societal significance, including an increased risk of $\mathrm{AD}$. Current evidence also supports relationships between SMI and the AD risk factors of increased depressive and anxiety symptoms as well as decreased cognitive, physical, and social activity. However, no research to date has: 1) elucidated mediators underlying linkages among SMI and memory decline; 2) characterized SMI subgroups at highest risk by identifying moderators of these mediational relationships; or 3) compared the differential predictive effects of specific SMI assessment items. This study addresses each of these critical knowledge gaps through the 
testing of our conceptual model using multilevel modeling in construct-level replication analyses [35, 36] across four longitudinal datasets.

\section{Conceptual framework}

SMI precipitates worry about AD [37, 38], depressive symptoms [39, 40], and withdrawal from cognitively protective activities (e.g., physical or social activities; [11]). These negative reactions to SMI represent one potential pathway by which SMI can contribute to AD development since both affective symptoms [41, 42] and reduced activity participation [43-46] are associated with increased AD risk. Furthermore, these potential reactions are also considered modifiable $\mathrm{AD}$ risk factors and opportunities for intervention to delay functional decline in older adults. The conceptual model guiding this study (Fig. 1) posits that, for some older adults, changes in modifiable AD risk factors mediate the relationship between SMI and objective memory decline. This model was developed based on three bodies of evidence: 1) SMI is associated with several modifiable AD risk factors, specifically increases in affective symptoms and reduced activity participation; 2) SMI is associated with an increased risk of objective memory decline; and, 3) the modifiable AD risk factors of interest in this study are associated with objective memory decline. Our conceptual model theorizes that negative reactions to SMI by high-risk older adults lead to an increase in modifiable AD risk factors (i.e., increased affective symptoms and reduced activity participation), subsequently leading to objective memory decline over time.

Additionally, our model considers individual differences that influence reactions to SMI, which is particularly important for identifying older adults at highest risk and ultimately tailoring intervention strategies. Higher neuroticism is consistently associated with SMI [4] and affective symptoms [47], and episodes of forgetfulness induce emotional distress and worry about developing AD in some older adults [48], particularly among those with a family history of $\mathrm{AD}[29,30]$. SMI predicts $\mathrm{AD}$ risk [49] and memory decline [50] for subgroups of older adults. Our conceptual model proposes that individual characteristics (e.g., personality and family history of $\mathrm{AD}$ ) influence older adults' reactions to SMI, and therefore moderate the relationship between SMI and modifiable AD risk factors. Due to the complex interplay of SMI and a chain of risk factors leading to AD, establishing moderators of these relationships will help us to determine susceptibility to negative reactions [51] and support interventions that focus on individual needs and risk profiles.

\section{Study aims}

The overall goals of this study are to characterize subgroups of older adults with SMI for whom these AD risk factors are more likely to develop over time, and to explicate longitudinal relationships among SMI, modifiable $\mathrm{AD}$ risk factors, and objective memory decline for subgroups of older adults. We will use construct-level replication analyses across multiple longitudinal datasets to investigate the following study aims:

Aim 1: Test the longitudinal predictive utility of SMI on modifiable AD risk factors.

Aim 2: Identify moderators of the relationships between SMI and modifiable AD risk factors.

Aim 3: Test a moderated mediation model where individual characteristics (e.g., neuroticism) moderate the relationship between SMI and modifiable AD risk

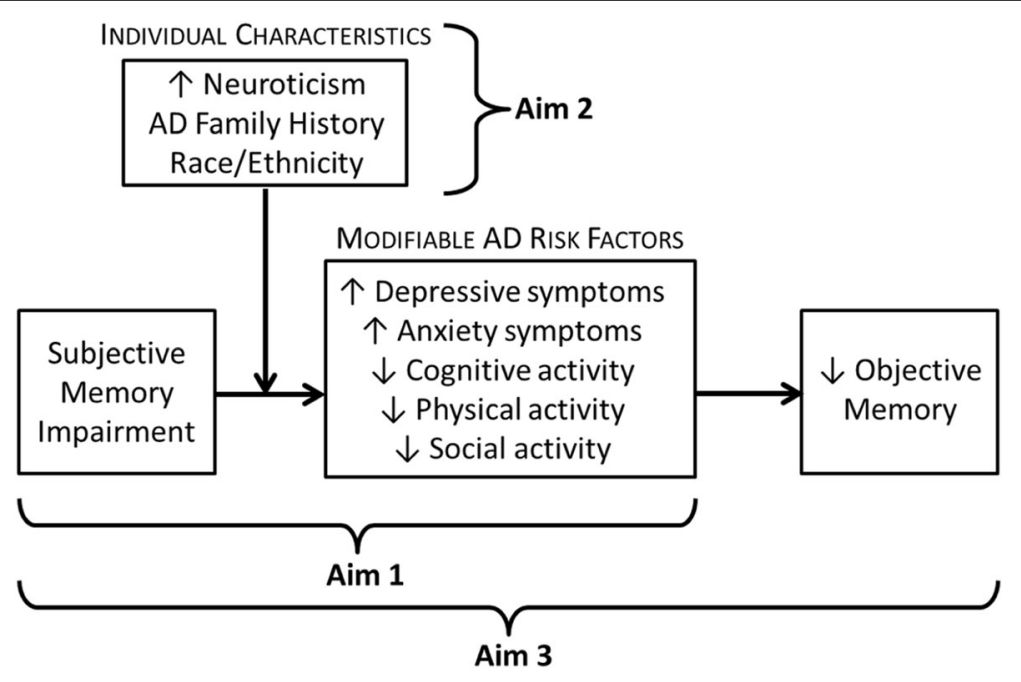

Fig. 1 Conceptual model guiding aims 
factors (e.g., depressive symptoms) that in turn mediate objective memory decline.

\section{Methods}

This study applies multilevel modeling (MLM) in construct-level replication analyses $[35,36]$ across four datasets collected in ongoing longitudinal studies of aging: Einstein Aging Study (EAS), Health and Retirement Study (HRS), Minority Aging Research Study (MARS), and National Health and Aging Trends Study (NHATS). These datasets represent a diverse group of over 40,000 adults (ages 55 and older) across follow-up periods of up to 22 years. As data collection intensive projects that obtain a wide variety of individual measures, data from longitudinal studies can be used to address questions about within-person processes as well as covariation, change, and between-person variability among these processes over time [36]. When carried out in large, diverse samples such as in the datasets in this study, we can examine developmental processes while also allowing for individual differences in these processes, rather than assuming equivalence in trajectories across all individuals. An additional advantage of this approach is to test the reproducibility of findings across multiple samples to establish the generalizability of the developed conceptual model.

\section{Sample characteristics}

Participants must meet the following basic criteria for inclusion in the study: age 55 or older, no clinical diagnosis of mild cognitive impairment or dementia at baseline (when available), and no objective memory impairment at baseline (when a diagnosis is not available). Brief descriptions of the samples and sampling strategy for each of the four studies are presented in Additional file 1: Table S1, including the approximate number of participants (total $n=47,253$ ) meeting the current study's basic inclusion criteria. These studies used a variety of sampling methodologies, including two large samples representative of the U.S. population of older adults (HRS and NHATS). MARS recruits exclusively African Americans, and EAS captures extensive follow up with the oldest old (85+ years) who are at greater risk for memory decline.

\section{Designs and procedures of selected studies}

The datasets for this project were collected within studies that are each uniquely relevant to achieving the study aims. EAS is a longitudinal cohort study examining cognitive aging, $\mathrm{AD}$, and other dementing disorders among community-dwelling older adults in an urban, multi-ethnic area of New York City [52]. Data collection began in 1993 and occurs annually via in-person comprehensive medical and neuropsychological examinations. HRS is a nationally representative longitudinal survey of individuals age 50 or older and provides a wealth of data on health, cognition, work and retirement, and psychosocial information [53]. Data collection began in 1992 and occurs biennially; samples are replenished every 6 years. As noted in Additional file 1: Table S1, the HRS sample is a multi-stage probability design that includes geographic stratification and oversampling of certain demographic groups. The survey is conducted as a mixed-mode design in both in-person and telephone formats, depending on timing (baseline or follow-up year) and age of participants. Since HRS includes participants aged 50 or older, we will limit our HRS dataset to ages 55 and older only. MARS is a longitudinal cohort study examining cognitive decline and $\mathrm{AD}$ in older African Americans exclusively in suburban and urban areas of Chicago, IL [54]. Data collection began in 2004 and occurs annually via in-person comprehensive clinical and neuropsychological examinations. Community engagement and relationship-building has been a key component of MARS recruitment, leading to a unique sample representative of African Americans with diverse demographic profiles. Similar to HRS, NHATS is meant to provide a national resource for investigating aging with the collection of data on physical and cognitive capacity, activities of daily life, and participation in valued activities [55]. Data collection began in 2011 and occurs annually via in-person interviews in a nationally representative sample of Medicare beneficiaries, with oversamples of older and Black individuals.

\section{Measures}

The selected datasets include one or more assessments of SMI, AD risk factors (affective symptoms and activity participation), individual characteristics of interest (personality, family history of $\mathrm{AD}$, race/ethnicity), and objective measures of memory performance. Additional file 2: Table S2 compares the instruments or measurement items available in each dataset for the concepts of interest in the current study. The heterogeneity of SMI measures across studies is a known limitation of existing research, and the selected datasets for this study are no exception. SMI measures utilized in this construct-level replication analysis include self-ratings of current memory performance (all four studies), comparisons to one's past memory performance over varying time periods (all four studies), comparison to others of the same age (one study), and rating of the interference of memory problems with important or daily activities (two studies).

\section{Analysis plan}

We will use MLM to determine whether higher levels of SMI are related to increased affective symptoms and decreased activity participation (Aim 1) and whether this relationship is moderated by neuroticism, family history of $\mathrm{AD}$, and race/ethnicity (Aim 2). Finally, we will test 
our full conceptual model that examines whether changes in affective symptoms and activity participation mediate the relationship between SMI and objective memory decline (Aim 3). Specifically, we will test moderated mediation as we hypothesize these relationships to hold among subgroups of older adults which will be identified in Aim 2.

\section{General approach to analysis}

Construct-level replication is a coordinated analytic approach that assumes consistency of construct representation across the datasets, rather than consistency of construct measurement required by stricter analytic techniques (e.g., mega-analysis). The selected datasets include measures that broadly reflect the constructs of interest for the current analyses though measured through different, standardized methods. Measures will be harmonized to the extent possible (e.g., similar scoring for items that are consistent across datasets) to allow for comparison of results and conclusions across datasets. However, a benefit to construct-level replication is the ability to probe differences that are primarily due to measurement; this is critical due to the differences across the datasets in the SMI measures used.

SMI items that rely on a person's perception of current memory ability tend to have stronger links to functional activities while SMI items that depend on an individual's memory-related concerns tend to have stronger links to affective symptoms [56, 57]. We hypothesize that items assessing current memory problems or current interference of memory problems with important activities reflect memory-related abilities (e.g., frequency of current memory problems), and that these items will be more strongly associated with changes in activity participation. In contrast, we hypothesize that items assessing memory change over time and items that ask for comparing memory performance to age-appropriate peers reflect ratings of memory concern (i.e., worry that memory has changed or is worse than peers) will be more strongly associated with changes in affective symptoms. We will specifically compare these different types of relationships within and across datasets as part of the analytic process.

\section{Other analytic considerations}

Similar data preparation techniques will be used across all datasets to facilitate comparisons consistent with the construct-level replication approach. For example, all datasets will use grand mean centering of continuous variables and consistent reference groups (e.g., males when sex is considered as a covariate) for categorical variables. We will use standardized coding for time (i.e., years) to equate the time metric across all datasets as well as calculate standardized estimates to compare the magnitude of effects across datasets. The longitudinal nature of the selected datasets will lead to some attrition over time. All participants will be included in analyses regardless of amount of follow up. Pattern mixture modeling will be used in sensitivity analyses to determine whether patterns of missingness impact substantive conclusions.

\section{Analytic approach for aim 1: Test the longitudinal predictive utility of SMI on modifiable $A D$ risk factors}

Using MLM we will first determine whether SMI, affective symptoms, and activity participation show significant within-person covariation over time. This analysis will determine whether at assessment occasions when an individual reports greater (compared to lesser) SMI severity, they also report greater affective symptoms and reduced activity participation. Next, we will test autoregressive MLMs. In these models, SMI from a previous occasion will be used to predict future modifiable $\mathrm{AD}$ risk factors of interest. We will also test whether these relationships are reciprocal by examining how affective symptoms (or activity participation) predict future reports of SMI. In these models, concurrent and lagged affective symptoms/activity participation (centered at baseline) will be used to predict SMI. These analyses will allow us to determine whether the relationships among SMI and the modifiable AD risk factors of interest are unidirectional.

\section{Analytic approach for aim 2: Identify moderators of the relationships between $S M I$ and modifiable $A D$ risk factors}

Extending the analyses described for Aim 1, we will add interactions with neuroticism, family history of $\mathrm{AD}$, and race/ethnicity in separate MLMs to determine whether these individual characteristics increase the severity of negative responses to SMI (i.e., the modifiable AD risk factors of interest: affective symptoms and activity participation). The moderators selected are between-person variables and will be entered as level 2 predictors in all equations tested.

\section{Analytic approach for aim 3: Test a moderated mediation model where individual characteristics (e.g., neuroticism) moderate the relationship between $S M I$ and modifiable $A D$ risk factors (e.g., depressive symptoms) that in turn mediate objective memory decline}

Finally, we will test moderated multilevel mediation [58]. As an example (Fig. 2), we examine the extent to which affective symptoms at time $t$ for individual $i$ account for the relationship between an individual's level of SMI at time $t$ and their objective memory decline over time. SMI and affective symptoms will be baseline-centered to track changes across time within an individual [59]. An 


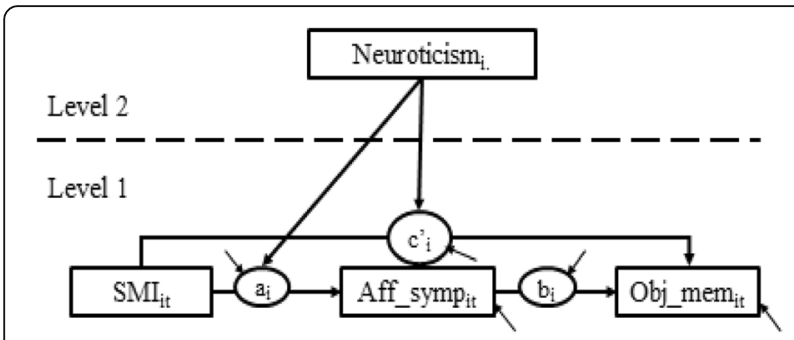

Fig. 2 Moderated multilevel mediation

individual's level of neuroticism is included as a moderator in the proposed moderated mediation in Fig. 2. We will test the significance of the c' path using methods suggested by Selig and Preacher [60]. This method will allow us to test the unique contributions of SMI and affective symptoms (or activity participation) to the prediction of objective memory performance.

\section{Discussion}

The earliest and most central deficit of AD is memory decline [61,62]. While some aspects of memory, such as procedural memory, are relatively preserved until later disease stages, episodic memory deficits are among the earliest signs of $\mathrm{AD}$ and are clinically relevant due to their functional implications [63]. Individuals with SMI are a heterogeneous group. Overall, they are at increased risk for memory decline [50] and $\mathrm{AD}$ [2], but most older adults with SMI will not go on to develop AD. Our understanding of the SMI-AD pathway is limited by a paucity of research on the temporal linkages among SMI, reactions to SMI associated with AD risk, and the earliest clinical indicator of AD: objective memory decline. Additionally, we lack knowledge regarding which individual characteristics (e.g., personality, family history of $\mathrm{AD})$ moderate the reactions to SMI that, in turn, mediate memory decline. This study employs an efficient, coordinated analytic approach to address these knowledge gaps and determine: 1 ) how SMI is associated with several modifiable AD risk factors (affective symptoms and activity participation) as well as declines in memory over time, and 2) how characteristics of individuals with SMI (i.e., personality, family history of $\mathrm{AD}$, and race/ethnicity) influence the strength of the relationships among SMI and the identified modifiable AD risk factors. In addition to explicating the complex relationships at play, identifying moderators of these relationships will allow characterization of highest risk SMI subgroups. This holds important implications for targeting future interventions, enriching samples for future research, and developing effective screening tools.

SMI is the focus of much current research, but these bodies of literature have remained disparate, linking SMI to $\mathrm{AD}$ or associating SMI with a variety of poor psychological or behavioral outcomes. There are few longitudinal examinations of these complex relationships in representative community-based samples. Replicating analyses across datasets will provide insight into the durability of the behavioral and psychological mechanisms (i.e., negative reactions to SMI) that increase AD risk among diverse groups of older adults with SMI. Furthermore, applying sophisticated analytic approaches and utilizing existing longitudinal datasets maximizes economy and efficiency.

At the population level, we are challenged with identifying risk profiles, screening tools, and intervention approaches that are applicable to a wide range of clinical settings and can be efficiently implemented in epidemiological research. Focusing on mediators of the relationship between SMI and memory decline over time will address scientific, as well as practical, needs in improving our understanding of preclinical AD. The potential mediators identified in this proposal are modifiable risk factors for $\mathrm{AD}$. Therefore, once we identify these as mediators of the pathway linking SMI to objective memory decline, interventions to prevent decline related to these risk factors can be developed, refined, and tested with specificity and clinical relevance in mind, ultimately combining this knowledge with developing evidence regarding the pathophysiology of AD.

\section{Additional files}

Additional file 1: Table S1. Sample Descriptions. (DOCX $18 \mathrm{~kb}$ )

Additional file 2: Table S2. Study Measures. (DOCX 20 kb)

\section{Abbreviations}

AD: Alzheimer's disease; EAS: Einstein Aging Study; HRS: Health and Retirement Study; MARS: Minority Aging Research Study; MLM: Multilevel Modeling; NHATS: National Health and Aging Trends Study; SMI: Subjective Memory Impairment

\section{Acknowledgements}

The authors thank Martin Sliwinski for his critical appraisal of the study proposal as well as the research teams and participants of the HRS, NHATS, EAS, and MARS studies.

\section{Funding}

This work was supported by the National Institute on Aging (NIA; grant number R01AG055398) to $\mathrm{NH}$. The funder has no role in study design, data collection, analysis, interpretation, or preparation of manuscripts. The current study uses data previously collected by four NIA-funded studies: HRS (grant number U01AG009740), NHATS (grant number U01AG32947), EAS (grant numbers R01AG12448, R01AG02672, \& AG003949), and MARS (grant numbers R01AG22018, R01AG17917, \& P30G10161).

\section{Availability of data and materials}

Two of the datasets analyzed in the current study are publicly available: HRS (http://hrsonline.isr.umich.edu/) and NHATS (https://www.nhats.org/). The EAS and MARS datasets are available from the Albert Einstein College of Medicine (https://www.einstein.yu.edu/departments/neurology/clinicalresearch-program/eas/data-sharing.aspx) and the Rush Alzheimer's Disease Center (https://www.radc.rush.edu/), respectively, but restrictions apply to the availability of these data. These datasets were used under license for the current study, and so are not publicly available. Data are however available 
from the authors upon reasonable request and with permission of the Principal Investigators of EAS and MARS as well as their affiliated organizations.

\section{Authors' contributions}

$\mathrm{NH}$ and JM conceptualized the study as well as drafted and revised the manuscript. Both authors read and approved the final manuscript.

\section{Ethics approval and consent to participate}

This study was approved by The Pennsylvania State University Institutional Review Board, referenced with the following study identifier: ID STUDY00007358. Informed consent was waived by the IRB at The Pennsylvania State University due to the exclusive use of secondary datasets.

\section{Consent for publication}

Not applicable.

\section{Competing interests}

The authors declare they have no competing interests.

\section{Publisher's Note}

Springer Nature remains neutral with regard to jurisdictional claims in published maps and institutional affiliations.

\section{Received: 27 September 2018 Accepted: 17 October 2018} Published online: 29 October 2018

\section{References}

1. Bäckman L, Jones S, Berger A-K, Laukka EJ, Small BJ. Cognitive impairment in preclinical Alzheimer's disease: a meta-analysis. Neuropsychology. 2005; 19(4):520-31.

2. Jessen F, Wolfsgruber S, Wiese B, Bickel H, Mösch E, Kaduszkiewicz H, et al. $\mathrm{AD}$ dementia risk in late $\mathrm{MCl}$, in early $\mathrm{MCl}$, and in subjective memory impairment. Alzheimers Dement. 2014;10:76-83.

3. Jessen $F$, Amariglio RE, van Boxtel M, Breteler M, Ceccaldi M, Chételat $G$, et al. A conceptual framework for research on subjective cognitive decline in preclinical Alzheimer's disease. Alzheimers Dement. 2014;10:844-52.

4. Reid LM, Maclullich AMJ. Subjective memory complaints and cognitive impairment in older people. Dement Geriatr Cogn Disord. 2006;22(5-6):471-85.

5. Saykin AJ, Wishart HA, Rabin LA, Santulli RB, Flashman LA, West JD, et al. Older adults with cognitive complaints show brain atrophy similar to that of amnestic MCl. Neurology. 2006;67:834-42.

6. Sperling RA, Aisen PS, Beckett LA, Bennett DA, Craft S, Fagan AM, et al. Toward defining the preclinical stages of Alzheimer's disease: recommendations from the National Institute on Aging-Alzheimer's association workgroups on diagnostic guidelines for Alzheimer's disease. Alzheimers Dement. 2011;7(3):280-92.

7. Eramudugolla R, Cherbuin N, Easteal S, Jorm AF, Anstey KJ. Self-reported cognitive decline on the informant questionnaire on cognitive decline in the elderly is associated with dementia, instrumental activities of daily living and depression but not longitudinal cognitive change. Dement Geriatr Cogn Disord. 2012;34(5-6):282-91.

8. Wang L, van Belle G, Crane PK, Kukull WA, Bowen JD, McCormick WC, et al. Subjective memory deterioration and future dementia in people aged 65 and older. J Am Geriatr Soc. 2004;52(12):2045-51.

9. Reisberg B, Shulman MB, Torossian C, Leng L, Zhu W. Outcome over seven years of healthy adults with and without subjective cognitive impairment. Alzheimers Dement. 2010:6:11-24.

10. Edmonds EC, Delano-Wood L, Galasko DR, Salmon DP, Bondi MW. Alzheimer's disease neuroimaging I. subjective cognitive complaints contribute to misdiagnosis of mild cognitive impairment. J Int Neuropsychol Soc. 2014;20(8):836-47.

11. Arenaza-Urquijo EM, Wirth M, Chételat G. Cognitive reserve and lifestyle: moving towards preclinical Alzheimer's disease. Front Aging Neurosci. 2015;7:134.

12. Ficker LJ, Lysack CL, Hanna M, Lichtenberg PA. Perceived cognitive impairment among African American elders: health and functiona impairments in daily life. Aging Ment Health. 2014;18(4):471-80.

13. Genziani M, Stewart $R$, Béjot $Y$, Amieva H, Artero S, Ritchie K. Subjective memory impairment, objective cognitive functioning and social activity in
French older people: findings from the three cities study. Geriatr Gerontol lnt. 2013:13(1):139-45.

14. Lee PL. The relationship between memory complaints, activity and perceived health status. Scand J Psychol. 2014;55(2):136-41.

15. Hill NL, Mogle J, Wion R, Munoz E, DePasquale N, Yevchak AM, et al. Subjective cognitive impairment and affective symptoms: a systematic review. The Gerontologist. 2016;56(6):e109-27.

16. Anstey K, Christensen H. Education, activity, health, blood pressure and apolipoprotein $\mathrm{E}$ as predictors of cognitive change in old age: a review. Gerontology. 2000;46:163-77.

17. Cooper C, Sommerlad A, Lyketsos CG, Livingston G. Modifiable predictors of dementia in mild cognitive impairment: a systematic review and metaanalysis. Am J Psychiatry. 2015;172(4):323-34.

18. Verghese J, Lipton RB, Katz MJ, Hall CB, Derby CA, Kuslansky G, et al. Leisure activities and the risk of dementia in the elderly. N Engl J Med Boston. 2003; 348(25):2508-16

19. Kaup AR, Byers AL, Falvey C, Simonsick EM, Satterfield S, Ayonayon HN, et al. Trajectories of depressive symptoms in older adults and risk of dementia. JAMA Psychiatry. 2016:73(5):525-31.

20. Lugtenburg A, Zuidersma M, Voshaar RC, Schoevers RA. Symptom dimensions of depression and 3-year incidence of dementia: results from the Amsterdam study of the elderly. J Geriatr Psychiatry Neurol. 2016;29:99-107.

21. Burmester B, Leathem J, Merrick P. Assessing subjective memory complaints: a comparison of spontaneous reports and structured questionnaire methods. Int Psychogeriatr. 2015;27:61-77.

22. Stern C, Munn Z. Cognitive leisure activities and their role in preventing dementia: a systematic review. Int J Evid Based Heal. 2010;8:2-17.

23. Russ TC, Hamer M, Stamatakis E, Starr JM, Batty GD. Psychological distress as a risk factor for dementia death. Arch Intern Med. 2011;171:1858-9.

24. Merema MR, Speelman CP, Foster JK, Kaczmarek EA. Neuroticism (not depressive symptoms) predicts memory complaints in some communitydwelling older adults. Am J Geriatr Psychiatry. 2013 Aug;21:729-36.

25. Pearman A, Storandt M. Predictors of subjective memory in older adults. Gerontol B Psychol Sci Soc Sci. 2004;59:P4-6.

26. Arbabi M, Zhand N, Eybpoosh S, Yazdi N, Ansari S, Ramezani M. Correlates of memory complaints and personality, depression, and anxiety in a memory clinic. Acta Med Iran. 2015:53:270-5.

27. Pearman A, Hertzog C, Gerstorf D. Little evidence for links between memory complaints and memory performance in very old age: longitudinal analyses from the Berlin aging study. Psychol Aging. 2014;29(4):828-42.

28. Comijs HC, Deeg DJ, Dik MG, Twisk JW, Jonker C. Memory complaints; the association with psycho-affective and health problems and the role of personality characteristics. A 6-year follow-up study. J Affect Disord. 2002;72: 157-65.

29. Ramakers $\mid H$, Visser PJ, Bittermann AJ, Ponds RW, van Boxtel MP, Verhey FR. Characteristics of help-seeking behaviour in subjects with subjective memory complaints at a memory clinic: a case-control study. Int J Geriatr Psychiatry. 2009:24(2):190-6.

30. Cantegreil-Kallen I, Pin S. Fear of Alzheimer's disease in the French population: impact of age and proximity to the disease. Int Psychogeriatr. 2012;24:108-16

31. Howell JC, Soyinka O, Parker M, Jarrett TL, Roberts DL, Dorbin CD, et al. Knowledge and attitudes in alzheimer's disease in a cohort of older African Americans and Caucasians. Am J Alzheimers Demen. 2016:31(4):361-7.

32. Cooper C, Tandy AR, Balamurali TB. Livingston G. a systematic review and meta-analysis of ethnic differences in use of dementia treatment, care, and research. Am J Geriatr Psychiatry. 2010;18(3):193-203.

33. Abdulrab K, Heun R. Subjective memory impairment. A review of its definitions indicates the need for a comprehensive set of standardised and validated criteria. Eur Psychiatry. 2008;23(5):321-30.

34. Rabin LA, Smart CM, Crane PK, Amariglio RE, Berman LM, Boada M, et al. Subjective cognitive decline in older adults: an overview of self-report measures used across 19 international research studies. J Alzheimers Dis. 2015;48(1):S63-86

35. Bauer DJ, Hussong AM. Psychometric approaches for developing commensurate measures across independent studies: traditional and new models. Psychol Methods. 2009:14(2):101-25.

36. Hofer SM, Piccinin AM. Integrative data analysis through coordination of measurement and analysis protocol across independent longitudinal studies. Psychol Methods. 2009;14(2):150-64. 
37. Kessler E-M, Bowen C, Baer M, Froelich L, Wahl H-W. Dementia worry: a psychological examination of an unexplored phenomenon. Eur J Ageing. 2012;9(4):275-84

38. Mol MEM, Ruiter RAC, Verhey FRJ, Dijkstra J, Jolles J. A study into the psychosocial determinants of perceived forgetfulness: implications for future interventions. Aging Ment Health. 2008;12(8):167-76.

39. Crane MK, Bogner HR, Brown GK, Gallo JJ. The link between depressive symptoms, negative cognitive bias and memory complaints in older adults. Aging Ment Health. 2007;11(6):708-15.

40. Pietrzak RH, Maruff P, Woodward M, Fredrickson J, Fredrickson A, Krystal JH, et al. Mild worry symptoms predict decline in learning and memory in healthy older adults: a 2-year prospective cohort study. Am J Geriatr Psychiatry. 2012;20(3):266-75.

41. Belleville S, Fouquet C, Duchesne S, Collins DL, Hudon C. Detecting early preclinical Alzheimer's disease via cognition, neuropsychiatry, and neuroimaging: qualitative review and recommendations for testing. J Alzheimers Dis. 2014;42(Suppl 4):S375-82.

42. Lopez OL, Jagust WJ, Dulberg C, Becker JT, DeKosky ST, Fitzpatrick A, et al. Risk factors for mild cognitive impairment in the cardiovascular health study cognition study: part 2. Arch Neurol. 2003;60(10):1394-9.

43. Dolcos S, MacDonald SW, Braslavsky A, Camicioli R, Dixon RA. Mild cognitive impairment is associated with selected functional markers: integrating concurrent, longitudinal, and stability effects. Neuropsychology. 2012;26(2):209-23.

44. Gold DA. An examination of instrumental activities of daily living assessment in older adults and mild cognitive impairment. J Clin Exp Neuropsychol. 2012;34(1):11-34

45. Reppermund S, Brodaty H, Crawford JD, Kochan NA, Draper B, Slavin MJ, et al. Impairment in instrumental activities of daily living with high cognitive demand is an early marker of mild cognitive impairment: the Sydney memory and ageing study. Psychol Med. 2013;43(11):2437-45.

46. Sachdev PS, Lipnicki DM, Crawford J, Reppermund S, Kochan NA, Trollor JN, et al. Risk profiles of subtypes of mild cognitive impairment: the Sydney memory and ageing study. J Am Geriatr Soc. 2012;60(1):24-33.

47. Lahey BB. Public health significance of neuroticism. Am Psychol. 2009;64(4):241-56.

48. French SL, Floyd M, Wilkins S, Osato S. The fear of alzheimer's disease scale: a new measure designed to assess anticipatory dementia in older adults. Int J Geriatr Psychiatry. 2012;27(5):521-8.

49. Jessen F, Wiese B, Bachmann C, Eifflaender-Gorfer S, Haller F, Kölsch H, et al. Prediction of dementia by subjective memory impairment. Arch Gen Psychiatry. 2010;67(4):414-22

50. Abner EL, Kryscio RJ, Caban-Holt AM, Schmitt FA. Baseline subjective memory complaints associate with increased risk of incident dementia: the PREADVISE trial. J Prev Alzheimers Dis. 2015;2(1):11-6.

51. Kraemer HC, Stice E, Kazdin A, Offord D, Kupfer D. How do risk factors work together? Mediators, moderators, and independent, overlapping, and proxy risk factors. Am J Psychiatry. 2001;158(6):848-56.

52. Katz MJ, Lipton RB, Hall CB, Zimmerman ME, Sanders AE, Verghese J, et al. Age-specific and sex-specific prevalence and incidence of mild cognitive impairment, dementia, and Alzheimer dementia in blacks and whites: a report from the Einstein aging study. Alzheimer Dis Assoc Disord. 2012; 26(4):335-43.

53. Sonnega A, Faul JD, Ofstedal MB, Langa KM, Phillips JW, Weir DR. Cohort profile: the health and retirement study (HRS). Int J Epidemiol. 2014;43(2): 576-85

54. Barnes LL, Shah RC, Aggarwal NT, Bennett DA, Schneider JA. The minority aging research study: ongoing efforts to obtain brain donation in African Americans without dementia. Curr Alzheimer Res. 2012;9(6):734-45.

55. Kasper J, Freedman V. National Health and Aging trends study (NHATS) user guide: rounds 1 - 7 beta release [internet]. Baltimore: Johns Hopkins University School of. Public Health. 2018; Available from: www.nhats.org

56. Fastame MC, Penna MP, Rossetti ES, Agus M. Perceived well-being and metacognitive efficiency in life course: a developmental perspective. Res Aging. 2013;35(6):736-49.

57. Marshall GA, Zoller AS, Lorius N, Amariglio RE, Locascio JJ, Johnson KA, et al. Functional activities questionnaire items that best discriminate and predict progression from clinically normal to mild cognitive impairment. Curr Alzheimer Res. 2015;12(5):493-502.

58. Bauer DJ, Preacher KJ, Gil KM. Conceptualizing and testing random indirect effects and moderated mediation in multilevel models: new procedures and recommendations. Psychol Methods. 2006;11(2):142-63.
59. Sliwinski MJ, Almeida DM, Smyth J, Stawski RS. Intraindividual change and variability in daily stress processes: findings from two measurement-burst diary studies. Psychol Aging. 2009;24(4):828-40.

60. Selig JP, Preacher KJ. Mediation models for longitudinal data in developmental research. Res Hum Dev. 2009;6(2-3):144-64.

61. Budson AE. Understanding memory dysfunction. Neurologist. 2009;15(2):71-9.

62. Kirova AM, Bays RB, Lagalwar S. Working memory and executive function decline across normal aging, mild cognitive impairment, and Alzheimer's disease. Biomed Res Int. 2015;2015(9):748212.

63. Gold CA, Budson AE. Memory loss in Alzheimer's disease: implications for development of therapeutics. Expert Rev Neurother. 2008:8:1879-91.

\section{Ready to submit your research? Choose BMC and benefit from:}

- fast, convenient online submission

- thorough peer review by experienced researchers in your field

- rapid publication on acceptance

- support for research data, including large and complex data types

- gold Open Access which fosters wider collaboration and increased citations

- maximum visibility for your research: over $100 \mathrm{M}$ website views per year

At $\mathrm{BMC}$, research is always in progress.

Learn more biomedcentral.com/submissions 